\title{
Competencias genéricas de los administradores de empresas
}

\author{
Sandra Patricia Rodríguez-Marmolejo ${ }^{1}$ \\ Corporación Universitaria Minuto de Dios - Buga \\ srodrigu159@uniminuto.edu.co \\ Yennifer Carolina Rojas-Arroyave ${ }^{2}$ \\ Corporación Universitaria Minuto de Dios - Buga \\ yrojasarroy@uniminuto.edu.co \\ Sigifredo Serna-Ospina ${ }^{3}$ \\ Corporación Universitaria Minuto de Dios - Buga \\ sernaos@uniminuto.edu.co
}

DOI: https://doi.org/10.21158/23227230.v9.n0.2019.2564

Cómo citar este artículo: Rodríguez-Marmolejo, S. P.; Rojas-Arroyave, Y. C.; Serna-Ospina, S. (2019). Competencias genéricas de los administradores de empresas. Revista Ploutos, 9, (Páginas). DOI: https://doi.org/10.21158/23227230.v9.n0.2019.2564

Fecha de recepción: 09 de abril de 2019

Fecha de aprobación: 04 de junio de 2019

\section{Resumen}

El objetivo de este artículo fue determinar las competencias genéricas de los administradores de empresas. Para esto se llevó a cabo un estudio con enfoque mixto haciendo uso de unidades muestrales localizadas en el municipio de Guadalajara de Buga y su zona de influencia, en tanto que es el contexto espacial donde se configuró el componente práctico de la investigación. La categorización de la muestra la conformaron académicos, estudiantes, empleadores y egresados, denominados «Grupo de consulta», representado por 207 individuos. La técnica seleccionada fue la encuesta y para el análisis estadístico se utilizó el programa SSPS Versión 23. Los resultados mostraron que se presentan diferencias significativas, especialmente entre el evaluado egresado vs. docentes y estudiantes vs. docentes, por lo que se infiere que los docentes en cuanto a las competencias genéricas tienen una apreciación con sentido académico, y los egresados y estudiantes tienen un concepto muy similar al sector productivo en los empleadores.

Palabras clave: administrador de empresas; competencias genéricas; competencias laborales; educación superior; identidad profesional; desarrollo profesional; entorno empresarial.

\footnotetext{
${ }^{1}$ Estudiante del programa de Administración de Empresas - Corporación Universitaria Minuto de Dios - Buga. ORCID https://orcid.org/0000-00024506-6818

${ }^{2}$ Estudiante del programa de Administración de Empresas - Corporación Universitaria Minuto de Dios - Buga. ORCID: https://orcid.org/0000$0002-6748-8691$

${ }^{3}$ Administrador de empresas de la Unidad Central Del Valle Del Cauca. Especialista en Gerencia Educativa con énfasis en formulación de proyectos - Universidad de Católica de Manizales. Magíster en Desarrollo Sostenible y Medio Ambiente - Universidad de Manizales. ORCID: https://orcid.org/0000-0002-7007-7375
} 


\title{
Generic competencies of business managers
}

\begin{abstract}
The aim of this article was to determine the generic competencies of business managers. For this purpose, the study was carried out with a mixed approach using sample units located in the municipality of Guadalajara de Buga and its area of influence, as this is the spatial context where the practical component of the research was configured. The categorization of the sample was made up of academics, students, employers, and graduates, and it was called the "Consultation Group", represented by 207 individuals. The technique used was the survey, and the statistical analysis was carried out with the SSPS program Version 23. The results showed that there are significant differences, especially among the evaluated graduate vs. teachers and students vs. teachers, so it is inferred that the appreciation that teachers have in terms of generic competencies has an academic sense, and the graduates and students have a concept that is very similar to the one that employers have in the productive sector.
\end{abstract}

Keywords: business administrator; generic competences; labor competences; higher education; professional identity; professional development; business environment.

Competências genéricas de administradores de empresas

\section{Resumo}

O objetivo deste artigo foi determinar as competências genéricas dos administradores de empresas. Para isso, foi realizado um estudo com abordagem mista, utilizando unidades amostrais localizadas no município de Guadalajara de Buga e sua área de influência, contexto espacial aonde aconteceu a pesquisa. A amostra foi categorizada por acadêmicos, estudantes, empregadores e graduados, denominada "Grupo de Consulta", representado por 207 indivíduos. A técnica selecionada foi a pesquisa e o programa SSPS Versão 23 foi utilizado para análise estatística. Os resultados mostraram que diferenças significativas estão presentes, principalmente entre os graduados avaliados vs. professores e alunos vs. professores, portanto, infere-se que os professores em termos de competências genéricas apreciam o senso acadêmico, e os graduados e os alunos têm um conceito muito semelhante ao setor produtivo dos empregadores.

Palavras-chave: administrador de empresas; competências genéricas; competências laborais; educação superior; identidade profissional; desenvolvimento profissional; ambiente empresarial.

\section{Compétences de base des dirigeants d'entreprises}

\section{Résumé}

L'objectif de cet article est de déterminer les compétences de base des dirigeants d'entreprise. Une étude de type approche mixte a donc été réalisée en utilisant des unités d'échantillonnage situées dans la municipalité de Guadalajara de Buga et sa zone d'influence dans la mesure où il s'agit de la zone gèographique où la composante pratique de l'investigation a été configurée. L'échantillon que l'on nomme "Groupe de consultation" est formè d'universitaires, étudiants, employeurs et diplômés et représente quelques 207 personnes. La technique de récupération des données utilisée est l'enquête de terrain et le programme SSPS version 23 a été utilisé pour l'analyse statistique. Les résultats montrent que des différences significatives sont présentes, en particulier entre les diplômés évalués et les enseignants, et entre étudiants et enseignants. Il est ainsi possible de déduire que les enseignants ont une appréciation des compétences de base au sens académique du terme alors que les diplômés et les étudiants ont un concept des compètences de base très similaire au secteur productif et aux employeurs.

Mots-clés: dirigeants d'entreprise; compétences de base; compétences professionnelles; enseignement supérieur; identité professionnelle; développement professionnel; environnement de travail. 


\section{Introducción}

Como parte de la dinámica y el amplio desarrollo del que goza actualmente el mundo productivo - de modo tal que supera los fenómenos que subyacen a los procesos de globalización en inserción de nuevas metodologías y políticas ambientales rígidas y eficaces - tanto los empresarios como las organizaciones deben enfrentar una serie de retos administrativos, económicos y sociales en aras de conservar su participación en los mercados de bienes y servicios cada vez más competitivos (Chiavenato, 2005). Por esta razón, todas las compañías, independientemente de su tamaño, deben contar con un recurso humano de alta calidad y competente que les permita redireccionar sus objetivos hacia las necesidades reales de mercado y, de este modo, lograr el cumplimiento de objetivos organizacionales y el alcance de su visión. En este sentido, deben tener en cuenta que es función del administrador, dentro de sus actividades, llevar a cabo una intervención eficiente para que las sociedades cumplan con dicho propósito (Villa y Poblete, 2011).

De este modo, se requiere que los procesos educativos en nivel superior cuenten con autonomía universitaria, entendida como la independencia de las instituciones de educación superior con respecto al entorno en el que se encuentran (Palmer, Montaño y Palou, 2009). Esta independencia debe ser de carácter político y administrativo, de manera que goce de competencias de autoevaluación y autogobierno (Garragori, 2007). Uno de los ejes transversales de la autonomía de la educación superior es la capacidad de elegir democráticamente sus dirigentes, así como la autoevaluación administrativa con procedimientos éticos que cubran la intervención y la participación de todos los integrantes de la comunidad académica. Lo anterior debe incluir a las directivas que son, en últimas, quienes deciden sobre los estatutos que regulan la institución y la diversidad académica que debe ofrecer a través de distintos programas dentro de la oferta académica (Plata, 2016).

Esta autonomía universitaria debe centrarse en tres factores determinantes: la autosuficiencia con base en los presupuestos, entendida como autarquía financiera; la prohibición a entidades de orden público de ingresar a las universidades sin previa autorización, reconocida con inviolabilidad del campus universitario; y las libres y 
democráticas elecciones dentro del campus donde se integran y participan tanto docentes como estudiantes en distintos grados, dependiendo del tipo de catedrático o de las condiciones contempladas en los manuales y las políticas que maneje la universidad (Plata, 2016).

De esta forma, al ser la universidad un ente independiente y autónomo, dentro de sus funciones deben fundamentarse prácticas que propendan al mejoramiento de los individuos a través de una formación integral en todos sus programas. Así mismo, los resultados y avances de esa formación de los egresados de las instituciones de educación superior deben responder al desarrollo logrado mediante las funciones básicas que haya desplegado la docencia, la investigación y la extensión que promueve el sector educativo (Delors, 1996). Por esta razón, en la última década, se han llevado a cabo la inserción y el desarrollo de iniciativas encaminadas por instituciones y organismos internacionales, como, por ejemplo, la Organización de las Naciones Unidas para la Educación, la Ciencia y la Cultura. La Unesco promueve e impulsa tendencias educativas a fin de que se contextualicen en los procesos de formación de distintos países y generen un impacto educativo mediante estrategias que deben ajustarse a los procesos de enseñanza-aprendizaje, en cuanto elemento integral y formal que permita cooperar con el desarrollo y el sostenimiento socioeducativo de las naciones (Unesco, 2009).

Una de estas intervenciones se llevó a cabo en Europa por medio de Tuning Educational Structures in Europe, un programa que se originó con el ánimo de establecer un área que integrara la educación superior con carácter socioeconómico. Esta iniciativa se centró en reflexionar sobre el futuro de la educación superior con relación al acelerado cambio y a la dinámica de la sociedad, así como a la urgencia de incluir patrones y factores de competitividad, compatibilidad y comparabilidad de una educación superior que ofrezca a los estudiantes la oportunidad de contar con fuentes de información fiable y objetiva acerca de la oferta de programas educativos, y a los empresarios hacer uso de herramientas y metodologías eficaces sobre lo que para ellos debería significar en la práctica un determinado título (Bravo, 2007). 
Este proyecto marcó una etapa definitiva para la educación superior y la real formación que deben recibir los futuros profesionales. Por tanto, su aceptación fue bastante significativa, pues no puso sus ojos en los programas de formación con un sentido unificado o estandarizado o en el título obtenido como tal, sino en identificar y establecer puntos en común que sirvieran de referentes para la estimulación, la comprensión y la convergencia dentro del proceso de enseñanza aprendizaje (González y Wagenaar, 2007). Con esta nueva mirada a la educación superior se incrementaron y facilitaron mejores formas de buscar y encontrar empleo en los países de la Unión Europea, el intercambio de estudiantes, la participación activa del Estado, de los estudiantes, de empresarios y de instituciones de educación superior, así como consensos de calidad de la formación académica y los contenidos educativos. De este modo, se abrió paso a la libertad, la autonomía y la diversidad.

El proyecto también se desarrolló en Latinoamérica en el 2004 y, posteriormente, en Colombia en el 2010. De esta manera, los países latinos también centraron su mirada en las competencias que debe tener un profesional integral y que pueda suplir las necesidades del mercado en una sociedad en constante cambio. Así, entonces, se logró que las universidades fortalecieran sus sistemas educativos, los programas de formación y las metodologías de enseñanza hacia un verdadero cambio productivo pensando en el potencial que deben desarrollar los egresados para ajustarse a los contextos del mercado, cada vez más exigente y competitivo.

En razón a lo anterior, el objetivo de esta investigación fue definir cuáles son las competencias genéricas que necesita el administrador de empresas para enfrentarse al mundo productivo.

\section{Fundamentación teórica}

\subsection{Modelo de educación superior}

El modelo clásico de educación superior ha experimentado ciertos cambios en los contextos internacional, regional y nacional que han incidido de forma directa en su conceptualización y dinámica en aras de establecer una mayor pertinencia. Estos cambios se 
basan, principalmente, en la masificación de la educación superior, la reducción del financiamiento estatal a procesos de formación académica superior, mayor control al gasto y a la inversión en el sector, el cambio de la autonomía universitaria a la heteronomía institucional, la responsabilidad social, el ajuste y la rendición de cuentas de la universidad y los sistemas pedagógicos tradicionales que han denotado la incapacidad para responder a las dinámicas y las necesidades actuales de la sociedad en la formación integral de sus individuos centrada en la creatividad, la participación, la comprensión y la construcción sociocultural del conocimiento (Unesco, 1998).

Además, las nuevas tecnologías de la información y la comunicación han variado e impactado de manera radical los procesos institucionales y la formación profesional, así como acrecentaron la cantidad de proveedores privados de procesos investigativos y educativos a nivel superior. Esto dio pie a una nueva redefinición de los saberes y fomentó el desplazamiento hacia la investigación como el motor de desarrollo en el cumplimiento de la función social de la universidad (Unesco, 1999).

Según la Unesco (1998), la búsqueda de soluciones a las distintas problemáticas derivadas de los procesos de globalización, polarización, democratización y marginación desencadenan una fragmentación dentro del contexto social sujeta, principalmente, a las formas de asumir la educación superior. De este modo, se exige de esta una reacción y una respuesta oportuna sujeta a los criterios de calidad, pertinencia e internacionalización. Así, la educación superior constituye una herramienta determinante en el propósito de lograr una cohesión social, integrar a los individuos pertenecientes a minorías, tanto urbanas como rurales, y fortalecer la identidad cultural, la diversidad y la protección del entorno. De ahí que se suscite su sentido sostenible para la humanidad, el establecimiento de un diálogo intercultural y la transición a una cultura de paz. 


\subsection{El entorno empresarial}

El entorno de la empresa hace referencia a los factores externos que se entienden como todo aquello que la rodea, desde las perspectivas socioeconómicas, tecnológicas, políticas, etc. Así mismo, a los factores internos, es decir, todo lo relacionado con el recurso humano, el cual lo conforman jefes y trabajadores, las relaciones laborales, la cultura organizacional, el clima organizacional, etc. De esta forma, en conjunto determinan los factores que inciden directa e indirectamente en la actividad de toda organización, los cuales, al retroalimentarse, afectan en mayor o menor grado la productividad (Barrios, 2011).

Durante mucho tiempo los factores internos fueron siempre el centro de atención primaria de las organizaciones y poca importancia se les dio a los factores externos. Sin embargo, dada la evolución y el desarrollo del enfoque de sistemas y contingencias, el entorno externo y el medio ambiente empiezan a exigir la importancia que ameritan, razón por la cual los empresarios empiezan a considerar variables socioeconómicas, políticas, culturales y tecnológicas como instrumentos y elementos determinantes para la toma de decisiones (Leandro, 2012).

De acuerdo con Cedeño (2005), el entorno empresarial obedece a la conjugación de distintos factores, lo que incluye los siguientes: las necesidades de los individuos, con el foco en los consumidores que son quienes desean pagar por satisfacer sus necesidades; los obstáculos para la empresa, lo que incluye acciones para competir en el mercado, así como los productos y los servicios de la competencia; y las exigencias y restricciones que toda organización debe atender. De igual manera, según este autor, a fin de que una organización subsista, crezca y se sostenga, sus actuaciones deben estar supeditadas a las amenazas, las oportunidades y la atención de las condiciones mínimas que el entorno exige, el cual se torna dinámico, complejo e inevitable, y lo conforman múltiples elementos que inciden en el actuar de la empresa de diversas formas. 
Estas formas se enmarcan en dos tipos de acciones. Las primeras, denominadas «directas», incluyen aquellos factores que inciden en el ambiente de la organización tales como la competencia, el consumidor, el sindicato, la mano de obra, los proveedores, la competencia, los accionistas y las entidades gubernamentales, entre otros.

Además, las acciones indirectas, referidas por Stoner, Freeman y Gilbert (2000) como aquellas variables de orden legal, político, económico, etc. Desde luego, resaltan que en cualquier momento una acción indirecta puede convertirse en directa y viceversa. De este modo, el ambiente de acción indirecta se compone de elementos que inciden en la atmósfera empresarial y en las operaciones organizacionales que pueden afectar a las empresas de dos modos:

1) Por medio de aquellos grupos externos sin ningún tipo de interés personal, pero que pueden influir de forma indirecta en la empresa mediante uno o más elementos contenidos en el ambiente de acción directa.

2) Aquellos elementos de acción indirecta condicionan el clima organizacional y en algún momento pueden responder.

Por tanto, los mismos autores resaltan la necesidad de especificar los componentes del ambiente de acción indirecta cuyas variables pueden ser las que se describen a continuación.

\subsubsection{Variables tecnológicas.}

Dado que la tecnología juega un papel determinante en la actualidad y, entre otros usos, sirve para determinar el tipo de servicios o productos que serán ofertados, la maquinaria y el equipo necesario, así como la manera como se administran las operaciones. Esto en especial en lo que se refiere al uso de elementos tecnológicos y de cómputo en las empresas con el fin de alcanzar nuevos niveles de posicionamiento competitivo en determinada industria. 


\subsubsection{Variables económicas.}

Las dinámicas dentro del sector económico pueden afectar de manera significativa las operaciones empresariales, por lo cual el crecimiento en dicho sector, las tasas de cambio, la inserción y el cumplimiento de políticas monetarias, fiscales y similares inciden sobre las actividades de la empresa a pesar de que hacen parte del ambiente de acción indirecta.

\subsubsection{Variables legales y políticas.}

La normatividad impuesta por el Estado para regular la actividad organizacional en muchos casos incentiva, pero en otros limita y hasta prohíbe, de tal modo que logra brindar confianza o no en la medida en que se establezcan las reglas.

\subsubsection{Variables socioculturales.}

Dependiendo de los valores que asuma una sociedad, el nivel de idiosincrasia, las costumbres, las tradiciones y los hábitos de una población específica influyen en buena medida en la manera como se deben llevar a cabo las operaciones en una empresa, pues determinan las relaciones interpersonales, la actitud laboral y la estructura organizacional, etc.

\subsubsection{Variables y aspectos internacionales.}

Muchos de los objetivos empresariales se centran en incursionar en el mercado internacional como una oportunidad de expansión, crecimiento y reconocimiento, de manera que deben atender a una debida evaluación del riesgo económico y político del país. Además, por esta razón debe ser importante reconocer los factores determinantes para la estabilidad y el equilibrio político y económico de los países.

\subsection{Competencias}

En América Latina se experimenta una transición hacia una nueva cultura emprendedora (Kantis, 2004). En Colombia se fomenta el emprendimiento desde hace más de 70 años (Varela, 2016) y, más aún, desde el nacimiento de la Ley 1014 de 2006 de fomento 
al emprendimiento. Así, las universidades e instituciones de educación en Colombia se han concebido como parte de los actores responsables de impulsar el desarrollo de capacidades y competencias. En este sentido han implementado actividades que tienen como objetivo promover una cultura de emprendimiento y de incentivo a prácticas efectivas en la administración, apoyadas en la ejecución de metodologías y programas que, de una manera u otra, ayudan a que los estudiantes desarrollen sus capacidades empresariales, con resultados incipientes con respecto al impacto económico y social que se persigue.

Ante esto, con el ánimo de propiciar espacios y herramientas que permitan identificar e intercambiar información para fortalecer la cooperación entre las instituciones de educación superior, fomentar la efectividad, la calidad y la transparencia, se llevó a cabo un proyecto que, hasta finales del 2004, era una experiencia única de la Unión Europea. Este, reconocido como Proyecto Tuning, hace partícipe a 175 universidades como respaldo y apoyo a una iniciativa de la zona que surgió en el 2001 a través de un arduo trabajo y en aras de crear un espacio de fortalecimiento para la educación superior ante el desafío que planteaba la Declaración de Bolonia, la cual señalaba una encrucijada en el desarrollo de la educación superior en Europa para reformar las estructuras de los sistemas de educación superior de manera convergente (Espacio Europeo de la Enseñanza Superior, 1999).

De este modo, se dio lugar a la implementación del proyecto Tuning en Latinoamérica 2004-2006, del que hicieron parte 180 instituciones de educación superior de 18 países latinos (Proyecto Alfa Tuning América Latina, 2007). En este sentido, los resultados obtenidos en el contexto regional fomentaron la implementación de dicho proyecto en Colombia en el 2010, en busca de proteger la amplia diversidad de la educación superior dentro del país y afianzar la autonomía universitaria de tal manera que fuera posible generar un reconocimiento más amplio y claro con respecto a la relevancia de desarrollar competencias en los administradores de empresas del país, así como en cada zona clasificada por la Asociación Colombiana de Facultades de Administración (Ascolfa, 2010) y con dicho panorama establecer una relación frente al contexto internacional. 
Uno de los criterios transversales que maneja el proyecto Tuning es el de competencias, el cual se define dentro de este como aquellas habilidades que le permiten al individuo conocer y comprender — teorías - , saber — modo de actuar- y adquirir valores — manera de percibir a los demás-. Lo anterior pone en evidencia cómo, además de en el conocimiento, se centra en estructurar al individuo como tal dentro de un contexto altamente competitivo, a lo que se hace alusión cuando se enuncia que el desarrollo de competencias permite el desempeño de los individuos en cuanto agentes responsables dentro de distintos contextos sociales y en distintas situaciones de la vida personal y social, de manera que aprenden a hacer, observar, actuar y disfrutar convenientemente evaluando alternativas, eligiendo las estrategias adecuadas y haciéndose cargo de las decisiones tomadas (Proyecto Alfa Tuning América Latina, 2007, p. 35).

Cabe resaltar cómo, dado que las competencias resultan la herramienta determinante bajo la cual subyace la estructuración y la formación de los sujetos, se obtienen dos grupos: en uno se encuentran unas competencias de carácter genérico que atienden a aquellos factores y elementos que se pueden compartir en cualquier entorno del saber -entre los que están la toma de decisiones, la capacidad de aprender y el diseño de proyectos, entre otrosy confieren a los sujetos un desempeño transversal que debería hacer parte de todo egresado de las instituciones de educación superior. En el otro se encuentran las competencias específicas, que incluyen intereses personales o particulares para el desarrollo efectivo de los conocimientos y las actitudes; estas competencias están íntimamente ligadas al perfil profesional, de modo que le brindan identidad y consistencia en cada campo disciplinar para optar a un título.

Para efectos de este artículo se consideran las competencias genéricas, teniendo en cuenta que, en el caso colombiano y según la Asociación Colombiana de Facultades de Administración o Ascolfa (2010), las universidades e instituciones de educación superior construyeron las competencias de los programas académicos de administración de empresas como parte de la contextualización de estas a la realidad y el entorno del país sin influencia de factores internacionales. 
Lo anterior, con consciencia de que el proceso de globalización en la actualidad se encuentra, entre otros elementos, condicionado por el aumento de la movilidad de los estudiantes y los profesionales - quienes exigen y necesitan información confiable y objetiva sobre los programas académicos que se ofrecen-, así como ligado a las necesidades e intereses de los empresarios dependiendo del contexto geográfico (Valero, 2011).

\section{Metodología}

La investigación tiene un enfoque mixto, el cual, según Hernández, Fernández y Baptista (2010), representa un conjunto de procesos sistemáticos, empíricos y críticos de investigación e implica la recolección y el análisis de datos cuantitativos y cualitativos, así como su integración y discusión conjunta, a fin de llegar a una integración de información que permita lograr un mayor entendimiento del fenómeno de investigación.

Las unidades muestrales se localizaron en el municipio de Guadalajara de Buga y su zona de influencia como contexto espacial donde se configuró el componente práctico de la investigación. La categorización de la muestra estuvo conformada por académicos, estudiantes, empleadores y egresados, denominados «Grupo de consulta» (Tabla 1); se representó a través de 207 individuos categorizados de acuerdo con el grupo de consulta al que pertenecen.

Tabla 1. Grupos de consulta

\begin{tabular}{|c|c|}
\hline Estrato & Muestra \\
\hline Empleadores & 80 \\
\hline Estudiantes & 90 \\
\hline Egresados & 22 \\
\hline Profesores & 15 \\
\hline Total & $\mathbf{2 0 7}$ \\
\hline
\end{tabular}

Fuente. Elaboración propia. 
La técnica seleccionada fue la encuesta. Se aplicó por medio de un cuestionario con el fin de obtener información susceptible de análisis sobre las competencias genéricas de los administradores de empresas del municipio de Guadalajara de Buga y su zona de influencia, las cuales se calificaron de acuerdo con la siguiente escala: neutral, algo importante, importante y muy importante. El estudio contiene pruebas en software SSPS versión 23, esto es, pruebas de normalidad, homocedasticidad, paramétricas y de concordancia.

Se clasifican las competencias genéricas de acuerdo con la media ponderada de la importancia y en conformidad con la apreciación de empleadores, egresados, estudiantes próximos a graduarse y docentes; en segundo lugar, se realiza una prueba de hipótesis para comprobar el nivel de importancia asociado a las 10 competencias genéricas más importantes.

Para el análisis estadístico en el programa SSPS, versión 23, se tuvieron en cuenta los siguientes conceptos: a) a partir de los grados de libertad el autor que se debía aplicar para la identificación de la normalidad de los datos-Kolmogorov-Smirnov o Shapiro-Wilk; y b) en la prueba de homocedasticidad -Supo, 3-, según el tipo de asimetría se determinó en la prueba de Levene que existía homocedasticidad o homogeneidad de varianza, de modo que se concluyó que las pruebas de aplicación debían ser paramétricas, usando prueba multivariada Anova-Tukey.

La presentación de los resultados está fundamentada en la obtención de la información recolectada de la aplicación del cuestionario. Para los efectos se analizaron los datos obtenidos en función de las diferentes unidades muestrales seleccionadas, en consideración a la relación sobre las competencias genéricas. El referido proceso consistió en una prueba de normalidad de los datos, por lo que se inició con las estadísticas de centramiento y dispersión de cada una de las variables medidas y su estadístico de contraste para verificar la normalidad (Triana, 2019). 


\section{Resultados}

En la tabla 2 se presenta el cruce de variables por categorías, por el cual se aprecia que, para el total de los grupos que conforman las unidades muestrales, las variables que se consideran determinantes con altos porcentajes de muy importante son liderazgo $75,36 \%$ - y trabajo en equipo - 75,84 \%-. Por el contrario, el resto de variables estudiadas resultaron percibidas como algo importante: emprendimiento -18,36\%-, empoderamiento $-18,84 \%$, compromiso $-17,87 \%$, sentido de pertenencia $25,6 \%$, identidad profesional -26,57\%-, desarrollo profesional -23,67\%- y responsabilidad social $-37,68 \%$ -

De igual manera, los hallazgos evidenciaron que resultan mucho más neutrales las siguientes categorías: empoderamiento -16,9\%-, compromiso - 27\%—, sentido de pertenencia $-26,1 \%-$, identidad profesional $-24,64 \%-$, desarrollo profesional $21,74 \%-$ y responsabilidad social -24,15\%-. En cuanto a las de valoración como importantes se tuvo desarrollo profesional -10,14 \%- y empoderamiento -3,86 \%-. 
Tabla 2. Tabla cruzada de valoración por categorías

\begin{tabular}{|c|c|c|c|c|c|c|c|}
\hline \multirow{3}{*}{\multicolumn{3}{|c|}{ Categorías }} & \multirow{2}{*}{\multicolumn{4}{|c|}{ Evaluado }} & \multirow{3}{*}{$\begin{array}{r}\text { Tota } \\
6 \\
\end{array}$} \\
\hline & & & & & & & \\
\hline & & & \multirow{2}{*}{$\begin{array}{c}\text { Empleadores } \\
5\end{array}$} & \multirow{2}{*}{$\begin{array}{c}\text { Egresados } \\
0\end{array}$} & \multirow{2}{*}{$\begin{array}{c}\text { Estudiantes } \\
1\end{array}$} & \multirow{2}{*}{$\begin{array}{c}\text { Profesor } \\
\text { es } \\
0\end{array}$} & \\
\hline \multirow[t]{5}{*}{ Liderazgo } & \multirow[t]{4}{*}{ Valoración } & Neutral & & & & & \\
\hline & & $\begin{array}{l}\text { Algo } \\
\text { importante }\end{array}$ & 4 & 12 & 2 & 2 & 20 \\
\hline & & Importante & 8 & 11 & 6 & 0 & 25 \\
\hline & & $\begin{array}{l}\text { Muy } \\
\text { importante }\end{array}$ & 63 & 67 & 13 & 13 & 156 \\
\hline & \multicolumn{2}{|l|}{ Total } & 80 & 90 & 22 & 15 & 207 \\
\hline \multirow{5}{*}{$\begin{array}{l}\text { Trabajo en } \\
\text { equipo }\end{array}$} & \multirow[t]{4}{*}{ Valoración } & Neutral & 0 & 0 & 2 & 0 & 2 \\
\hline & & $\begin{array}{l}\text { Algo } \\
\text { importante }\end{array}$ & 0 & 9 & 2 & 0 & 11 \\
\hline & & Importante & 24 & 7 & 2 & 4 & 37 \\
\hline & & $\begin{array}{l}\text { Muy } \\
\text { importante }\end{array}$ & 56 & 74 & 16 & 11 & 157 \\
\hline & \multicolumn{2}{|l|}{ Total } & 80 & 90 & 22 & 15 & 207 \\
\hline \multirow{5}{*}{$\begin{array}{l}\text { Emprendi } \\
\text { miento }\end{array}$} & \multirow[t]{4}{*}{ Valoración } & Neutral & 9 & 9 & 2 & 0 & 20 \\
\hline & & $\begin{array}{l}\text { Algo } \\
\text { importante }\end{array}$ & 8 & 22 & 4 & 4 & 38 \\
\hline & & Importante & 43 & 34 & 9 & 4 & 90 \\
\hline & & $\begin{array}{l}\text { Muy } \\
\text { importante }\end{array}$ & 20 & 25 & 7 & 7 & 59 \\
\hline & \multicolumn{2}{|l|}{ Total } & 80 & 90 & 22 & 15 & 207 \\
\hline \multirow{5}{*}{$\begin{array}{l}\text { Toma de } \\
\text { decisiones }\end{array}$} & \multirow[t]{4}{*}{ Valoración } & Neutral & 14 & 10 & 0 & 0 & 24 \\
\hline & & $\begin{array}{l}\text { Algo } \\
\text { importante }\end{array}$ & 16 & 0 & 1 & 0 & 17 \\
\hline & & Importante & 0 & 2 & 4 & 0 & 6 \\
\hline & & $\begin{array}{l}\text { Muy } \\
\text { importante }\end{array}$ & 50 & 78 & 17 & 15 & 160 \\
\hline & \multicolumn{2}{|l|}{ Total } & 80 & 90 & 22 & 15 & 207 \\
\hline \multirow[t]{6}{*}{$\begin{array}{l}\text { Empodera } \\
\text { miento }\end{array}$} & \multirow[t]{5}{*}{ Valoración } & $\begin{array}{l}\text { Poco } \\
\text { importante }\end{array}$ & 8 & 0 & 0 & 0 & 8 \\
\hline & & Neutral & 10 & 19 & 6 & 0 & 35 \\
\hline & & $\begin{array}{l}\text { Algo } \\
\text { importante }\end{array}$ & 13 & 20 & 6 & 0 & 39 \\
\hline & & Importante & 35 & 28 & 4 & 6 & 73 \\
\hline & & $\begin{array}{l}\text { Muy } \\
\text { importante }\end{array}$ & 14 & 23 & 6 & 9 & 52 \\
\hline & \multicolumn{2}{|l|}{ Total } & 80 & 90 & 22 & 15 & 207 \\
\hline \multirow{5}{*}{$\begin{array}{l}\text { Compromi } \\
\text { so }\end{array}$} & \multirow[t]{4}{*}{ Valoración } & Neutral & 15 & 31 & 10 & 0 & 56 \\
\hline & & $\begin{array}{l}\text { Algo } \\
\text { importante }\end{array}$ & 6 & 20 & 5 & 6 & 37 \\
\hline & & Importante & 13 & 32 & 7 & 9 & 61 \\
\hline & & $\begin{array}{l}\text { Muy } \\
\text { importante }\end{array}$ & 46 & 7 & 0 & 0 & 23 \\
\hline & \multicolumn{2}{|l|}{ Total } & 80 & 90 & 22 & 15 & 207 \\
\hline & Valoración & Neutral & 7 & 38 & 9 & 0 & 54 \\
\hline $\begin{array}{l}\text { pertenenci } \\
\text { a }\end{array}$ & & $\begin{array}{l}\text { Algo } \\
\text { importante }\end{array}$ & 9 & 34 & 8 & 2 & 53 \\
\hline & & Importante & 25 & 11 & 5 & 2 & 43 \\
\hline & & $\begin{array}{l}\text { Muy } \\
\text { importante }\end{array}$ & 39 & 7 & 0 & 11 & 57 \\
\hline & Total & & 80 & 90 & 22 & 15 & 207 \\
\hline & Valoración & Neutral & 12 & 26 & 8 & 5 & 51 \\
\hline
\end{tabular}




\begin{tabular}{|c|c|c|c|c|c|c|c|}
\hline \multirow{4}{*}{$\begin{array}{l}\text { Identidad } \\
\text { profesiona } \\
\text { l }\end{array}$} & & $\begin{array}{l}\text { Algo } \\
\text { importante }\end{array}$ & 15 & 31 & 6 & 3 & 55 \\
\hline & & Importante & 31 & 21 & 6 & 4 & 62 \\
\hline & & $\begin{array}{l}\text { Muy } \\
\text { importante }\end{array}$ & 22 & 12 & 2 & 3 & 39 \\
\hline & \multicolumn{2}{|l|}{ Total } & 80 & 90 & 22 & 15 & 207 \\
\hline \multirow{6}{*}{$\begin{array}{l}\text { Desarrollo } \\
\text { profesiona } \\
\text { l }\end{array}$} & \multirow[t]{5}{*}{ Valoración } & $\begin{array}{l}\text { Poco } \\
\text { importante }\end{array}$ & 0 & 21 & 0 & 0 & 21 \\
\hline & & Neutral & 15 & 16 & 10 & 4 & 45 \\
\hline & & $\begin{array}{l}\text { Algo } \\
\text { importante }\end{array}$ & 16 & 23 & 7 & 3 & 49 \\
\hline & & Importante & 29 & 21 & 4 & 3 & 57 \\
\hline & & $\begin{array}{l}\text { Muy } \\
\text { importante }\end{array}$ & 20 & 9 & 1 & 5 & 35 \\
\hline & \multicolumn{2}{|l|}{ Total } & 80 & 90 & 22 & 15 & 207 \\
\hline \multirow{5}{*}{$\begin{array}{l}\text { Responsa } \\
\text { bilidad } \\
\text { Social }\end{array}$} & \multirow[t]{4}{*}{ Valoración } & Neutral & 14 & 30 & 6 & 0 & 50 \\
\hline & & $\begin{array}{l}\text { Algo } \\
\text { importante }\end{array}$ & 34 & 29 & 9 & 6 & 78 \\
\hline & & Importante & 18 & 22 & 4 & 2 & 46 \\
\hline & & $\begin{array}{l}\text { Muy } \\
\text { importante }\end{array}$ & 14 & 9 & 3 & 7 & 33 \\
\hline & \multicolumn{2}{|l|}{ Total } & 80 & 90 & 22 & 15 & 207 \\
\hline \multirow[t]{6}{*}{ Total } & \multirow[t]{5}{*}{ Valoración } & $\begin{array}{l}\text { Poco } \\
\text { importante }\end{array}$ & 8 & 21 & 0 & 0 & 29 \\
\hline & & Neutral & 101 & 179 & 54 & 9 & 343 \\
\hline & & $\begin{array}{l}\text { Algo } \\
\text { importante }\end{array}$ & 121 & 200 & 50 & 26 & 397 \\
\hline & & Importante & 226 & 189 & 51 & 34 & 500 \\
\hline & & $\begin{array}{l}\text { Muy } \\
\text { importante }\end{array}$ & 344 & 311 & 65 & 81 & 801 \\
\hline & \multicolumn{2}{|l|}{ Total } & 800 & 900 & 220 & 150 & 2070 \\
\hline
\end{tabular}

Fuente. Elaboración propia.

En la tabla 3 se presenta la prueba de normalidad mediante el establecimiento de hipótesis y la determinación — según los grados de libertad— de qué autor o prueba se debía seguir; en este caso fue Shapiro-Wilk, lo cual generó como resultado que no existía un comportamiento normal en la distribución de los encuestados y las escalas de valoración del modelo encuesta - poco importante, neutral, algo importante, importante y muy importante-

De este modo se obtienen las hipótesis:

- Ho: existe un comportamiento normal en la distribución de los encuestados y la escala de neutral, algo importante, importante y muy importante.

- Hi: no existe un comportamiento normal en la distribución de los encuestados y la escala de neutral, algo importante, importante y muy importante. 
Tabla 3. Prueba de normalidad

\begin{tabular}{|l|c|c|c|c|c|c|}
\hline \multirow{2}{*}{ Encuestado } & \multicolumn{3}{|c|}{ Kolmogorov-Smirnov } & \multicolumn{3}{c|}{ Shapiro-Wilk } \\
\cline { 2 - 8 } & Estadístico & gl & Sig. & Estadístico & gl & Sig. \\
\hline Empleadores & 0,265 & 36 & 0 & 0,784 & 36 & 0 \\
\hline Egresados & 0,278 & 32 & 0 & 0,863 & 32 & 0,001 \\
\hline Estudiantes & 0,226 & 32 & 0 & 0,88 & 32 & 0,002 \\
\hline Profesores & 0,24 & 40 & 0 & 0,8 & 40 & 0 \\
\hline
\end{tabular}

Fuente. Elaboración propia.

Por tanto, se acepta la Hi, lo que significa que no existe un comportamiento normal en la distribución de los encuestados y la escala de los valores neutral, algo importante, importante y muy importante.

Así mismo, se aplicó la prueba de homogeneidad de varianza apoyada en Levene al formular hipótesis para una valoración de significancia del 5 \% y determinar que «sí existía homocedasticidad entre las varianzas de los encuestados». Esto a partir de la prueba de normalidad y homogeneidad de varianza, por la cual se concluye que las pruebas a aplicar son paramétricas, dado que en este estudio, al tener un planteamiento multivariado, se aplica la prueba Anova-Tukey, lo que revela que en el estudio no existía una igualdad significativa entre la media de los grupos encuestados, esto es, empleadores, egresados, estudiantes y docentes.

Se concluye que existían diferencias significativas entre el evaluado egresado vs. docentes y estudiantes vs. docentes, lo que indica cómo los docentes en las competencias genéricas tienen una apreciación con sentido académico mientras los egresados y los estudiantes tienen un concepto muy similar al sector productivo en los empleadores (Figura 1). 
Figura 1. Media de escala de los encuestados

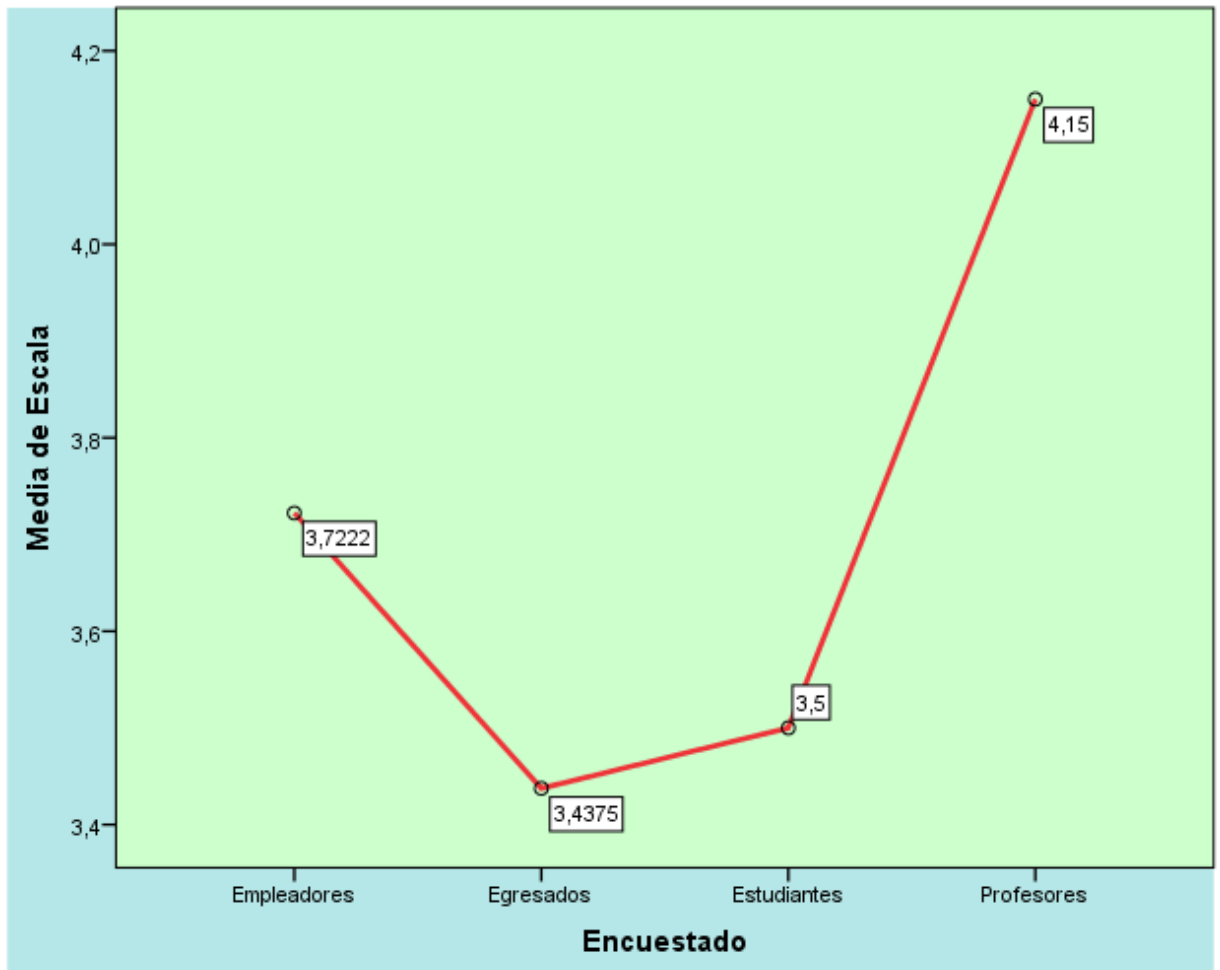

Fuente. Elaboración propia.

Para la concordancia entre los evaluados se aplicó la prueba Kappa de Cohen, teniendo presente una interpretación en la escala Landis y Koch (1977). De allí se tiene que, significativamente, tienen relación los empleadores con los egresados, con una fuerza de concordancia de forma moderada, y entre los egresados y estudiantes en proceso de grado presentan el mismo sentido anterior de relación y concordancia (Tablas 4 y 5). En consecuencia, se plantea, en términos estadísticos, la siguiente prueba de hipótesis de concordancia, con la finalidad de evaluar y comparar el nivel de significancia del $5 \%$ o 0,05 y así determinar si se acepta la hipótesis nula o la alterna:

- Ho: no existe concordancia significativa de los evaluados empleadores y los egresados.

- Hi: existe concordancia significativa de los evaluados empleadores y los egresados. 
Por lo anterior, al relacionar la significación aproximada de 0,004 es menor al nivel de significancia de 0,05, aceptando la hipótesis alterna que enuncia «existe concordancia significativa de los evaluados empleadores y los egresados».

Tabla 4. Prueba de concordancia empleadores vs. egresados

\begin{tabular}{|c|c|c|c|c|c|}
\hline Empleadores & & & & & \\
\hline \multicolumn{6}{|c|}{ Medidas simétricas } \\
\hline \multicolumn{2}{|l|}{ Egresados } & Valor & $\begin{array}{c}\text { Error } \\
\text { estandarizado } \\
\text { asintótico }^{\mathrm{a}}\end{array}$ & $\begin{array}{c}\mathrm{T} \\
\text { aproximadab }^{\mathrm{b}}\end{array}$ & $\begin{array}{c}\text { Significación } \\
\text { aproximada }\end{array}$ \\
\hline $\begin{array}{l}\text { Medida de } \\
\text { acuerdo }\end{array}$ & Kappa & 0,545 & 0,198 & 2,886 & 0,004 \\
\hline \multicolumn{2}{|c|}{$\mathrm{N}$ de casos válidos } & 10 & & & \\
\hline \multicolumn{6}{|c|}{ a. No se presupone la hipótesis nula. } \\
\hline \multicolumn{6}{|c|}{ b. Utilización del error estándar asintótico que presupone la hipótesis nula. } \\
\hline
\end{tabular}

Fuente. Elaboración propia.

Tabla 5. Prueba de concordancia egresados vs. estudiantes

\begin{tabular}{|c|c|c|c|c|c|}
\hline Egresados & & & & & \\
\hline \multicolumn{6}{|c|}{ Medidas simétricas } \\
\hline \multicolumn{2}{|c|}{ Estudiantes } & Valor & $\begin{array}{c}\text { Error } \\
\text { estandarizado } \\
\text { asintótico }^{\mathrm{a}}\end{array}$ & $\begin{array}{c}\mathrm{T} \\
\text { aproximada }^{\mathrm{b}}\end{array}$ & $\begin{array}{c}\text { Significación } \\
\text { aproximada }\end{array}$ \\
\hline $\begin{array}{l}\text { Medida } \\
\text { de } \\
\text { acuerdo }\end{array}$ & Kappa & 0,167 & 0,157 & 0,972 & 0,331 \\
\hline \multicolumn{2}{|c|}{$\mathrm{N}$ de casos válidos } & 10 & & & \\
\hline \multicolumn{6}{|c|}{ a. No se presupone la hipótesis nula. } \\
\hline
\end{tabular}

Fuente. Elaboración propia.

Las hipótesis fueron:

- Ho: no existe concordancia significativa del evaluado empleadores y los estudiantes.

- Hi: existe concordancia significativa del evaluado empleadores y los estudiantes. 
Se acepta Ho: no existe concordancia significativa del evaluado, los empleadores y los estudiantes, y su coeficiente de concordancia de Kappa de Cohen según la escala de Fleiss es leve. Cabe destacar que la aplicación de concordancia entre los otros encuestados que arrojó el programa SSPS versión 23 indicó que no existía una significación de importancia, por tanto, su fuerza de concordancia según Landis y Kock (Tabla 6) estaría definida por un sentido muy pobre-bajo. El nivel de significancia por debajo del $5 \%$ se mira en el nivel de concordancia.

Tabla 6. Valoración del coeficiente de Kappa

\begin{tabular}{ll}
\hline $\begin{array}{l}\text { Coeficiente } \\
\text { kappa }\end{array}$ & Fuerza de la concordancia \\
\hline 0,00 & Pobre (Poor) \\
$0,01 \cdot 0,20$ & Leve (Slight) \\
$0,21 \cdot 0,40$ & Aceptable (Fair) \\
$0,41 \cdot 0.60$ & Moderada (Moderate) \\
$0,61 \cdot 0,80$ & Considerable (Substantial) \\
$0,81 \cdot 1.00$ & Casi perfecta (Almost perfect) \\
\hline
\end{tabular}

Fuente. Landis y Koch, 1977.

Según las competencias genéricas para los empleadores, egresados, estudiantes y docentes evaluados, se visualiza, a través de un informe descriptivo (Tabla 7) que contiene medidas de tendencia central y dispersión, cómo en los empleadores se puede inferir que la percepción de esas competencias representa ser algo importante, según una buena proporción de los empleadores que afirman lo anterior, pues el coeficiente de variación ilustra una homogeneidad del 18,84\%.

Con respecto a la asimetría se refleja un comportamiento positivo que detona la importancia de la medida de tendencia central - media - con respecto a lo que refleja el $50 \%$ de los datos de los empleadores - mediana-. Cabe destacar que los empleadores reflejan un comportamiento platicurtico - Chata- con respecto a la curtosis de los datos, lo cual indica que una buena proporción de los encuestados tienen concentración en una percepción entre algo importante y muy importante en las competencias genéricas evaluadas. 
Tabla 7. Medidas de tendencia central y dispersión en empleadores

\begin{tabular}{|c|c|c|c|c|}
\hline \multicolumn{5}{|c|}{ Descriptivo } \\
\hline \multicolumn{3}{|l|}{ Encuestado } & Estadístico & Error \\
\hline \multirow[t]{13}{*}{ Empleadores } & \multicolumn{2}{|l|}{ Media } & 3,72 & 0,117 \\
\hline & \multirow{2}{*}{$\begin{array}{l}95 \% \text { de intervalo } \\
\text { de confianza para } \\
\text { la media }\end{array}$} & $\begin{array}{l}\text { Límite } \\
\text { inferior }\end{array}$ & 3,48 & \\
\hline & & $\begin{array}{l}\text { Límite } \\
\text { superior }\end{array}$ & 3,96 & \\
\hline & \multicolumn{2}{|c|}{ Media recortada al $5 \%$} & 3,69 & \\
\hline & \multicolumn{2}{|l|}{ Mediana } & 4 & \\
\hline & \multicolumn{2}{|l|}{ Varianza } & 0,492 & \\
\hline & \multicolumn{2}{|l|}{ Desviación estándar } & 0,701 & \\
\hline & \multicolumn{2}{|l|}{ Mínimo } & 3 & \\
\hline & \multicolumn{2}{|l|}{ Máximo } & 5 & \\
\hline & \multicolumn{2}{|l|}{ Rango } & 2 & \\
\hline & \multicolumn{2}{|l|}{ Rango intercuartil } & 1 & \\
\hline & \multicolumn{2}{|l|}{ Asimetría } & 0,449 & 0,393 \\
\hline & \multicolumn{2}{|l|}{ Curtosis } & $-0,823$ & 0,768 \\
\hline
\end{tabular}

Fuente. Elaboración propia.

En los egresados reflejan un promedio de autopercepción con dicha categoría de ser algo importante, con un comportamiento homogéneo según el coeficiente de variación del $26,56 \%$. Así mismo, su incidencia asimétrica es positiva, resaltando la importancia que tiene la media con respecto al 50 \% de los evaluados egresados - mediana- y una curtosis con comportamiento platicurtico, con concentración entre la escala neutral y muy importante (Tabla 8). 
Tabla 8. Medidas de tendencia central y dispersión en egresados

\begin{tabular}{|c|c|c|c|c|}
\hline \multicolumn{5}{|c|}{ Descriptivo } \\
\hline \multicolumn{3}{|c|}{ Encuestado } & \multirow{2}{*}{$\begin{array}{c}\text { Estadístico } \\
3,44\end{array}$} & \multirow{2}{*}{$\begin{array}{c}\begin{array}{c}\text { Error } \\
\text { estándar }\end{array} \\
0,162\end{array}$} \\
\hline \multirow[t]{13}{*}{ Egresados } & \multicolumn{2}{|l|}{ Media } & & \\
\hline & \multirow[t]{2}{*}{$\begin{array}{l}95 \% \text { de intervalo de } \\
\text { confianza para la } \\
\text { media }\end{array}$} & $\begin{array}{l}\text { Límite } \\
\text { inferior }\end{array}$ & 3,11 & \\
\hline & & $\begin{array}{l}\text { Límite } \\
\text { superior }\end{array}$ & 3,77 & \\
\hline & \multicolumn{2}{|l|}{ Media recortada al $5 \%$} & 3,43 & \\
\hline & \multicolumn{2}{|l|}{ Mediana } & 3 & \\
\hline & \multicolumn{2}{|l|}{ Varianza } & 0,835 & \\
\hline & \multicolumn{2}{|l|}{ Desviación estándar } & 0,914 & \\
\hline & \multicolumn{2}{|l|}{ Mínimo } & 2 & \\
\hline & \multicolumn{2}{|l|}{ Máximo } & 5 & \\
\hline & \multicolumn{2}{|l|}{ Rango } & 3 & \\
\hline & \multicolumn{2}{|l|}{ Rango intercuartil } & 1 & \\
\hline & \multicolumn{2}{|l|}{ Asimetría } & 0,332 & 0,414 \\
\hline & \multicolumn{2}{|l|}{ Curtosis } & $-0,603$ & 0,809 \\
\hline
\end{tabular}

Fuente. Elaboración propia.

El comportamiento de análisis en los estudiantes con respecto a las medidas de tendencia central aporta en las escalas de valoración un sentir de algo importante en las competencias genéricas, según la homogeneidad de los datos medido con el coeficiente de variación del 28,11 \%. Su comportamiento de deformación genera mayor valor en el 50\% de los estudiantes.

El comportamiento de apuntamiento de la percepción de los estudiantes representa un comportamiento platicurtico con tendencias en la escala de valoración entre neutral y muy importante, alejándose de la percepción de respuesta algo importante en las presentes competencias genéricas analizadas (Tabla 9). 
Tabla 9. Medidas de tendencia central y dispersión en estudiantes

\begin{tabular}{|c|c|c|c|c|}
\hline \multicolumn{5}{|c|}{ Descriptivo } \\
\hline \multicolumn{3}{|l|}{ Encuestado } & Estadístico & Error \\
\hline \multirow[t]{13}{*}{ Estudiantes } & \multicolumn{2}{|l|}{ Media } & 3,5 & 0,174 \\
\hline & \multirow{2}{*}{$\begin{array}{l}95 \% \text { de } \\
\text { intervalo de } \\
\text { confianza para } \\
\text { la media }\end{array}$} & $\begin{array}{l}\text { Límite } \\
\text { inferior }\end{array}$ & 3,15 & \\
\hline & & $\begin{array}{l}\text { Límite } \\
\text { superior }\end{array}$ & 3,85 & \\
\hline & \multicolumn{2}{|c|}{ Media recortada al 5\% } & 3,5 & \\
\hline & \multicolumn{2}{|l|}{ Mediana } & 4 & \\
\hline & \multicolumn{2}{|l|}{ Varianza } & 0,968 & \\
\hline & \multicolumn{2}{|c|}{ Desviación estándar } & 0,984 & \\
\hline & \multicolumn{2}{|l|}{ Mínimo } & 2 & \\
\hline & \multicolumn{2}{|l|}{ Máximo } & 5 & \\
\hline & \multicolumn{2}{|l|}{ Rango } & 3 & \\
\hline & \multicolumn{2}{|c|}{ Rango intercuartil } & 1 & \\
\hline & \multicolumn{2}{|l|}{ Asimetría } & $-0,108$ & 0,414 \\
\hline & \multicolumn{2}{|l|}{ Curtosis } & $-0,931$ & 0,809 \\
\hline
\end{tabular}

Fuente. Elaboración propia.

Con respecto al sentir de los docentes se evidencia una percepción promedio de ser importante al tener como referente una homogeneidad representada en el 18,55 \% de coeficiente de variación. Así mismo, la dispersión a partir de la deformación y el apuntamiento reflejan en la percepción de los docentes un sentir de importante en el 50 \% de los docentes evaluados, con un comportamiento platicurtico que va entre la percepción de algo importante y muy importante (Tabla 10). 
Tabla 10. Medidas de tendencia central y dispersión en docentes

\begin{tabular}{|c|c|c|c|c|}
\hline \multicolumn{5}{|c|}{ Descriptivo } \\
\hline \multicolumn{3}{|c|}{ Encuestado } & Estadístico & Error \\
\hline \multirow[t]{13}{*}{ Docentes } & \multicolumn{2}{|l|}{ Media } & 4,15 & 0,122 \\
\hline & \multirow[t]{2}{*}{$\begin{array}{l}95 \% \text { de intervalo de } \\
\text { confianza para la media }\end{array}$} & $\begin{array}{l}\text { Límite } \\
\text { inferior }\end{array}$ & 3,9 & \\
\hline & & $\begin{array}{l}\text { Límite } \\
\text { superior }\end{array}$ & 4,4 & \\
\hline & \multicolumn{2}{|l|}{ Media recortada al $5 \%$} & 4,17 & \\
\hline & \multicolumn{2}{|l|}{ Mediana } & 4 & \\
\hline & \multicolumn{2}{|l|}{ Varianza } & 0,592 & \\
\hline & \multicolumn{2}{|l|}{ Desviación estándar } & 0,77 & \\
\hline & \multicolumn{2}{|l|}{ Mínimo } & 3 & \\
\hline & \multicolumn{2}{|l|}{ Máximo } & 5 & \\
\hline & \multicolumn{2}{|l|}{ Rango } & 2 & \\
\hline & \multicolumn{2}{|l|}{ Rango intercuartil } & 1 & \\
\hline & \multicolumn{2}{|l|}{ Asimetría } & $-0,268$ & 0,374 \\
\hline & \multicolumn{2}{|l|}{ Curtosis } & $-1,235$ & 0,733 \\
\hline
\end{tabular}

Fuente. Elaboración propia.

\section{Conclusiones}

Según los datos y los hallazgos obtenidos se evidencia que, con respecto a las competencias genéricas de los evaluados, se tiene una percepción muy homogénea en el sentir de ser algo importante y muy importante, lo que refleja un comportamiento de valoración como necesidad formativa en dichas competencias.

De los grupos encuestados —empleadores, egresados, estudiantes y docentes - se presentan diferencias significativas, en especial entre el evaluado egresado vs. docentes y estudiantes vs. docentes, de lo que se infiere que los docentes, en las competencias genéricas, tienen una apreciación con sentido académico, mientras los egresados y los estudiantes tienen un concepto muy similar al del sector productivo en los empleadores. Este hecho indica la tendencia de las nuevas generaciones de profesionales hacia la construcción de una realidad productiva bajo la cual centran su formación, pues si bien es cierto que el proceso académico es determinante para la formación del estudiante, debe llevarse a cabo bajo 
nuevas formas y paradigmas encaminados a que el conocimiento tenga un sentido didáctico y práctico, y no solo se centre en brindar y recibir conocimiento teórico.

El liderazgo, el trabajo en equipo y la toma de decisiones son las competencias genéricas que resultan de mayor relevancia para los grupos encuestados, pues definen la labor del administrador de empresas, si se tiene en cuenta que los nuevos jefes y administradores buscan potencializar su labor en el mercado a través de la toma de decisiones asertivas que involucren a todos los colaboradores en el mejoramiento continuo de sus actividades y funciones, y que denote un liderazgo participativo a través de distintos métodos actualizados como el benchmarking, la lluvia de ideas y los seguimientos individuales y colectivos, entre otros. Esto, con el fin de fomentar un trabajo en equipo en el que se concentran las necesidades recíprocas como una oportunidad para insertar las habilidades y los conocimientos basada en la confianza y crear así seguridad en el cumplimiento de los objetivos organizacionales. El hecho de que sean estas tres competencias genéricas las de mayor importancia en la percepción de los encuestados constituye una oportunidad de crecimiento individual en el alcance de objetivos colectivos por los que todos aportan y todos ganan haciendo uso de la integración, la cooperación y la capacidad de entrega.

Otro de los hallazgos relevantes del estudio expone que en cuanto a competencias genéricas consideradas como algo importantes o neutrales se encuentran las siguientes: el emprendimiento, el empoderamiento, el compromiso, el sentido de pertenencia, la identidad profesional, el desarrollo profesional y la responsabilidad social. Esto indica que se carece de un interés por parte de los grupos encuestados en fomentar una formación e intervención integral del administrador de empresas, pues si es cierto que la toma de decisiones y el trabajo en equipo son determinantes y transversales dentro de cualquier proceso productivo, también lo son el llevar a cabo una función con sentido de pertenencia y ética profesional que efectivice la relación entre el equipo de trabajo y la organización. 
Competencias tales como el emprendimiento, el empoderamiento y el sentido de pertenencia con la profesión y con la sociedad exigen de todo un proceso organizado que requiere, a su vez, del desarrollo de una serie de actividades puntuales que le permitan al administrador de empresas ejecutar de manera adecuada la materialización de su objetivo como empresario, en tanto este cuente con las condiciones, los conocimientos, la capacidad y la habilidad para explotar una idea que agregue valor a la sociedad y resuelva o mejore una problemática existente. Además, se debe reconocer que aquellas ideas de negocio que se planifican y ejecutan con efectividad constituyen una de las alternativas más rentables en la disminución de las altas tasas de desempleo que atraviesan muchos países fomentando el crecimiento de la economía, la competitividad en los distintos sectores productivos y mejorando las condiciones de vida del tejido social.

\section{Referencias}

Ascolfa (Asociación Colombiana de Facultades de Administración). (2010). Las competencias de los administradores en Colombia a la luz del proyecto Tuning América Latina. Bogotá: Ascolfa.

Barrios, V. (2011, noviembre 30). Ambiente externo de las organizaciones. Recuperado de https://bit.ly/340VPLN

Bravo, N. (julio de 2007). Competencias proyecto Tuning-Europa, Tuning-América Latina. Bogotá, Colombia. Recuperado de https://bit.ly/340BaHF

Cedeño, A. (2005). Administración de la empresa (3aㅡ ed.). Costa Rica: Editorial Universidad Estatal a Distancia.

Chiavenato, I. (2005). Introducción a la administración. Colombia: Editorial McGraw-Hill.

Delors, J. (1996). La educación encierra un tesoro. Madrid: Ediciones Unesco.

Espacio Europeo de la Enseñanza Superior. (19 de junio de 1999). Declaración de Bolonia. Bolonia, Italia. Recuperado de https://bit.ly/3oxITXn

Garagorri, X. (2007). Currículum basado en competencias: aproximación al estado de la cuestión. Aula de Innovación Educativa, (161), 47- 55. Recuperado de https://bit.ly/2T2964t

González, J.; Wagenaar, R. (2007). Tuning educational structures in Europe. Informe final del proyecto piloto: Fase II. Bilbao: Universidad de Deusto, Universidad de Groningen. Recuperado de https://bit.ly/3yAp0DI 
Hernández, R.; Fernández, C.; Baptista, P. (2010). Metodología de la investigación (5ª ed.). McGrawHill.

Kantis, H. (2004). Desarrollo emprendedor: América Latina y la experiencia internacional. Nueva York: Banco Interamericano de Desarrollo.

Landis, J. R.; Koch, G. G. (1977). The measurement of observer agreement for categorical data. Biometrics, 33, 159-174. D0I: https://doi.org/10.2307/2529310

Leandro, G. (2012). El entorno de la organización. Aula de Economía. Recuperado de https://bit.ly/2RqQriz

Palmer, A.; Montaño, J.; Palou, M. (2009). Las competencias genéricas en la educación superior: estudio comparativo entre la opinión de empleadores y académicos. Psicothema, 21(3), 433438. Recuperado de https://bit.ly/3bJEw6l

Plata, J. (2016). Breves aportes a la discusión sobre la autonomía universitaria en Colombia. Nuevos Paradigmas de las Ciencias Sociales Latinoamericanas, VII(14), 209-224. Recuperado de https://bit.ly/3u5AX06

Proyecto Alfa Tuning América Latina. (Febrero de 2007). Tuning América Latina. Conclusiones y propuestas. Ciudad de México, México. Recuperado de https://bit.ly/3frz0WM

Stoner, J.; Freeman, R.; Gilbert, D. (2000). Administración (6ª ed.). México: Prentice Hall.

Triana, L. (2019). Datos estadísticos. Guadalajara de Buga: Uniminuto.

Unesco. (1998). Conferencia Mundial sobre la Educación. La educación superior en siglo XXI. Visión y acción. París: Unesco. Recuperado de https://bit.ly/3hHg0q4

Unesco. (31 de agosto de 1999). Conferencia Mundial sobre la Educación Superior en el Siglo XXI: Visión y Acción. Memorias. París, Francia. Recuperado de https://bit.ly/3hHdBMb

Unesco. (2009). Conferencia mundial sobre educación superior. Las nuevas dinámicas de la educación superior y de la investigación para el cambio social y el desarrollo. París: Ediciones Unesco.

Valero, G. (2011). Las competencias de los administradores en Colombia a la luz del proyecto Tuning. Caso capítulo oriente. Puente Revista Científica, 5(1). Recuperado de https://bit.ly/3hHzNpE

Varela, R. O. (2016). Colombia small-and medium-sized enterprise's 70 years of progress: what's next? Small Enterprise Research, 23(3), 302-315. DOI: https://doi.org/10.1080/13215906.2016.1269241

Villa, A.; Poblete, M. (2011). Evaluación de competencias genéricas: principios, oportunidades y limitaciones. Bordón, 63(1), 147-170. Recuperado de https://bit.ly/3u5Bb7U 